\title{
Synthesis of New Pyrazolones and Fused Pyrazole Derivatives as Antimicrobial Agents
}

\author{
Eman M. Kamel, S.T. Gaballah, Y.M.A. Mohamed ${ }^{*}$ and \\ Nadia R. Mohamed*** \\ Photochemistry Department, National Research Center, Giza, \\ Egypt, Department of Pharmaceutical Chemistry, School of \\ Pharmacy, University of Oslo, Oslo, P.O. Box 1068 Blindern, \\ N-0316, Oslo, Norway and ${ }^{* *}$ Chemistry Department, El-Aflaj \\ Girls College, El-Kharj University, Kingdom of Saudi Arabia.
}

\begin{abstract}
QME new pyrazolone and fused pyrazole derivatives start D from enaminone-type precursor. This approach constitutes a novel and advantageous alternative for the synthesis of the target heterocycles. Thus, the reaction of pyrazolone 1 and DMFDMA afforded 4-(dimeth-ylamino)methylidenepyrazol-3-one (2). Enaminone 2 was utilized as a key intermediate for the synthesis of new pyrazolones $8,9,12$, and 14 , fused pyrazolones $4-7$, and 10 and the spiro adducts $15 \mathrm{a}, \mathrm{b}$. The antimicrobial activity of some of the newly synthesized adducts were evaluated against six strains of Gram positive, Gram negative and fungi in comparison with Ampicillin and Nystatine.
\end{abstract}

Keywords: Pyrazolones, Pyrazoloisoxazole, Pyrazolopyrazole, Pyrazoloquinoline, Pyrazoloenaminone, Antimicrobial activity.

Pyrazole and its derivatives are potential building blocks in synthesis of new $\operatorname{drugs}^{(1-3)}$. They show broad spectrum biological activities such as antiinflammatory $^{(4-7)}$, antitumor ${ }^{(8-10)}$, and antimicrobial ${ }^{(11,12)}$. Most recently, pyrazolone derivatives showed high inhibition activity against Mycobacterium tuberculosis (MTB), the causative agent of tuberculosis ${ }^{(13)}$. Pyrazoles also show potent inhibition against monoamine oxidase-B (MOA-B) ${ }^{(14)}$, human telomerase ${ }^{(15)}$, and dependent kinase $(\mathrm{CDK})^{(16)}$. A new pyrazolone derivative known as edaravone (3-methyl-1-phenyl-2-pyrazoline-5-one) is being tested as a drug in clinical practice for brain ischemia ${ }^{(17,18)}$. It was also found to be effective against myocardial ischemia ${ }^{(19)}$. Pyrazolone derivatives such as antipyrine, aminopyrine and dipyrone are known as antipyretic and analgesic substances ${ }^{(20)}$ and their pharmacological mechanism has been widely investigated. Some of the aryl pyrazole derivatives are useful in the treatment of a variety of disorders caused by Human Immunodeficiency Virus (HIV) and other genetic diseases caused by retroviruses such as Acquired Immune Deficiency Syndrome (AIDS) ${ }^{(21)}$. In the same context, substituted and fused pyrazoles are considered as the structural motif of many commercial drugs such as Zometapine ${ }^{(22)}$, Celebrex ${ }^{(23)}$, Sildenafil ${ }^{(24)}$, and Rimonabant ${ }^{(25)}$. Pyrazoles also exhibit diverse properties ${ }^{(26)}$ and useful as

Corresponding author. E-mail nadia_ragab_m@yahoo.com 
starting materials for the synthesis of other fused heterocycles ${ }^{(27,28)}$. In addition to their higher biological activities, pyrazoles are also used for the preparation of dyes $^{(29)}$ couplers for photographic materials ${ }^{(30)}$ and as herbicides ${ }^{(31-33)}$.

There is continuous medical need for new anti-infective therapies due to the global emergence of resistance to antimicrobial agents which is increasingly limiting the effectiveness of current drugs. This motivated us to synthesize some new pyrazole and fused pyrazole derivatives hoping that they show potential antimicrobial activity. Depending on all the previous information and in continuation to our previous work on synthesizing biologically active nitrogencontaining heterocycles ${ }^{(34,35)}$, we report the use of 4-(dimethylamino) methylidenepyrazol-3-one as a precursor for the synthesis of the new compounds reported herein.

\section{Results and Discussion}

\section{Chemistry}

The reaction of active methylene-containing compound with dimethylformamide dimethyl acetal (DMFDMA) is the general and the most prevalent method to synthesize enaminones ${ }^{(36)}$. Thus, 5-methyl-2,4-dihydro-pyrazol-3-one $(1)^{(37)}$ was treated with DMFDMA in boiling DMF to afford a mixture of the pyrazoloenamine 2 in $80 \%$ yield in addition to the unexpected pyrazolodimethylaminoethylidenepyrazol-3-one (3) in 20\% yield in a molar ratio 4:1 (Scheme 1). A reasonable explanation of the formation of the adduct 3 could be attributed to the intermolecular interaction between two molecules of 2 followed by the loss of dimethylamine under the reaction conditions.

The structure of the two products 2 and 3 was elucidated on the basis of the analytical and spectral data. The mass spectrum of the products revealed molecular ion peaks at $\mathrm{m} / \mathrm{z}=153$ and $261\left(\mathrm{M}^{+}\right)$, respectively. The ${ }^{1} \mathrm{H}-\mathrm{NMR}$ of compound 3 showed a singlet at $\delta=2.21 \mathrm{ppm}$ corresponding to the $\mathrm{NMe} 2$ group, two singlets at $\delta=2.73$ and $2.89 \mathrm{ppm}$ for the two $\mathrm{CH}_{3}$ groups attached to the pyrazolone ring, singlet at $\delta=6.04 \mathrm{ppm}$ for the ethylidene proton and a broad $\mathrm{D}_{2} \mathrm{O}$-exchangeable signal at $\delta=12.29 \mathrm{ppm}$ for the two $\mathrm{NH}$ groups. The IR and ${ }^{13} \mathrm{C}$-NMR spectrum were in agreement with both structures.

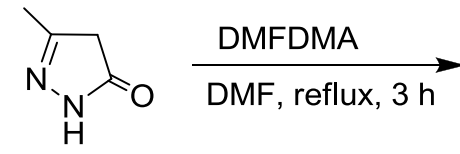

1

Egypt. J. Chem. 53, No. 5 (2010)<smiles>CNCC(C=C1C(=O)NN=C1C)=C1C(=O)NN=C1C</smiles>

Scheme 1 
Next, we focused our attention on the synthesis of pyrazolone and fused pyrazole derivatives utilizing pyrazoloenamine 2 as a key precursor. As shown in Scheme 2, compound 2 was treated with hydroxylamine hydrochloride to yield the corresponding pyrazolo[4,3-d]isoxazole 4 in $75 \%$ yield. The formation of the isoxazole adducts upon the interaction between enaminone and hydroxylamine hydrochloride has been reported previously ${ }^{(38-40)}$. The structure of 4 was elucidated by the analytical and spectral data. It is noteworthy to mention here that the synthesis of 4-methyl-6H-pyrazolo[4,3-d]isoxazole (4) was claimed before by the reaction of 5-chloro-3-methyl-1H-pyrazole-4-carbaldehyde with hydroxylamine ${ }^{(41-43)}$. However, these reports are mystic since neither experimental procedure nor spectroscopic data of the reaction product was provided $^{(44)}$. Interaction of 2 with hydrazine hydrate in ethanol containing acetic acid afforded pyrazolo[3,4-c]pyrazole 5 in $78 \%$ yield. The formation of pyrazole ring on treatment of enaminone with hydrazine hydrate has been investigated previously ${ }^{(38,40,45)}$. In addition, treatment of 2 with thiourea yielded the pyrazolopyrimidinethiole adducts 7 . The $1 \mathrm{H}-\mathrm{NMR}$ of the latter compound revealed a singlet at $\delta=1.90 \mathrm{ppm}$ for the $\mathrm{SH}$ proton beside the other characteristic signals of the structure. Compound 8 was formed via the interaction of 2 with phenylisothiocyanate in ethanol containing $\mathrm{NaOH}$. The $1 \mathrm{H}-$ NMR spectrum supported the existence of the NMe2 as a singlet at $\delta=2.33 \mathrm{ppm}$ beside the other characteristic signals.

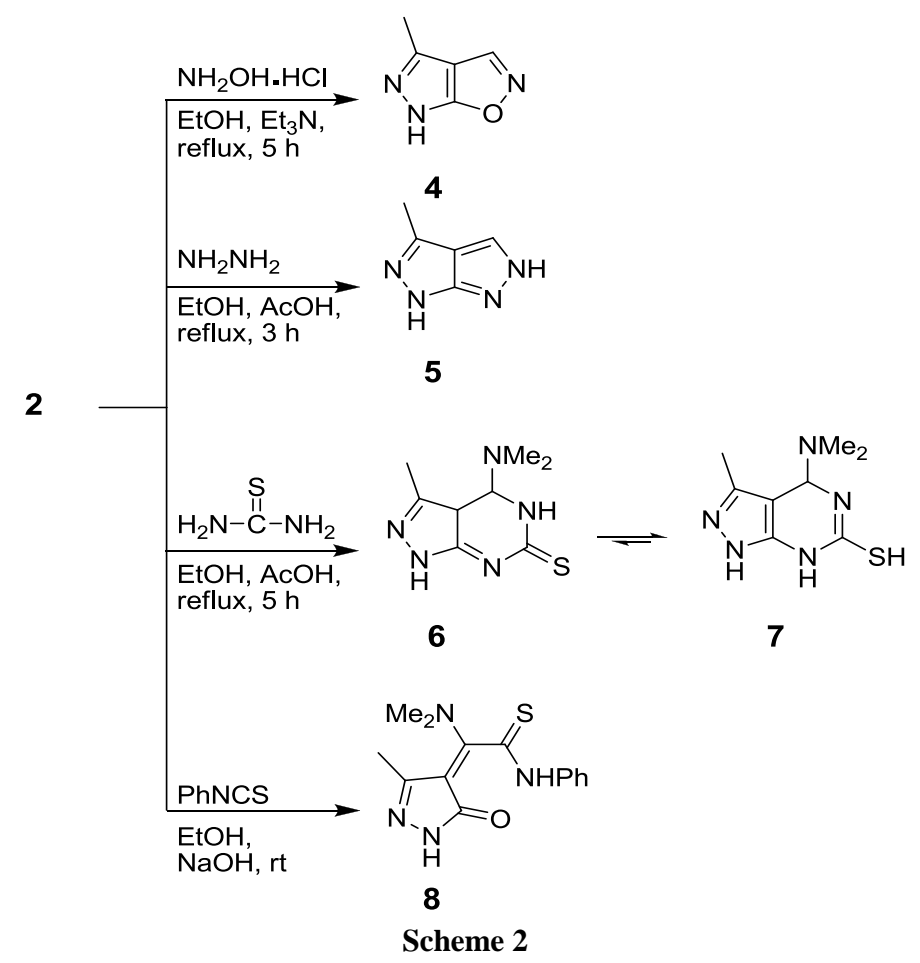

Egypt. J. Chem. 53, No. 5 (2010) 
Scheme 3 outlines the reaction of 2 with aminothiouracil and $\alpha$-naphthylamine. Pyrazoloenamine 2 was reacted with an equivalent amount of aminothiouracil in refluxing ethanol in presence of acetic acid to afford 9, however, the reaction time was long (16 hr) and the yield was poor (13\%). To improve the yield and shorten the reaction time, the reaction was repeated by adopting MORE [Microwave-induced Organic Reactions Enhancement] technology invented by Bose $^{(46,47)}$. Thus, the reaction of 2 with aminothiouracil could be completed in microwave oven using small amount of dimethylfor-mamide within $10 \mathrm{~min}$. The yield of the product was also improved to $58 \%$. The structure of 9 was confirmed by $1 \mathrm{H}-\mathrm{NMR}$ which revealed the characteristic signals at $\delta=2.09$ and $2.51 \mathrm{ppm}$ for $\mathrm{CH} 3$ and $\mathrm{NMe} 2$ groups, a singlet at $\delta=5.04 \mathrm{ppm}$ for the $\mathrm{CH}$ of the pyridine fused ring, in addition to the signals of $4 \mathrm{NH}$ groups. Shorter reaction time (2 $\mathrm{min})$ of pyrazoloenamine 2 and aminothiouracil under microwave irradiation afforded the open adduct 10 .

We also attempted to synthesize compound 11 via the reaction between 2 and $\alpha$-naphthylamine in refluxing ethanol in presence of acetic acid. Yet, this reaction did not yield the desired product, but instead transamination took place to afford adduct 12 in $69 \%$ yield. All the microanalytical and spectroscopic data were in accordance with the structure of compound 12 (Scheme 3 ).

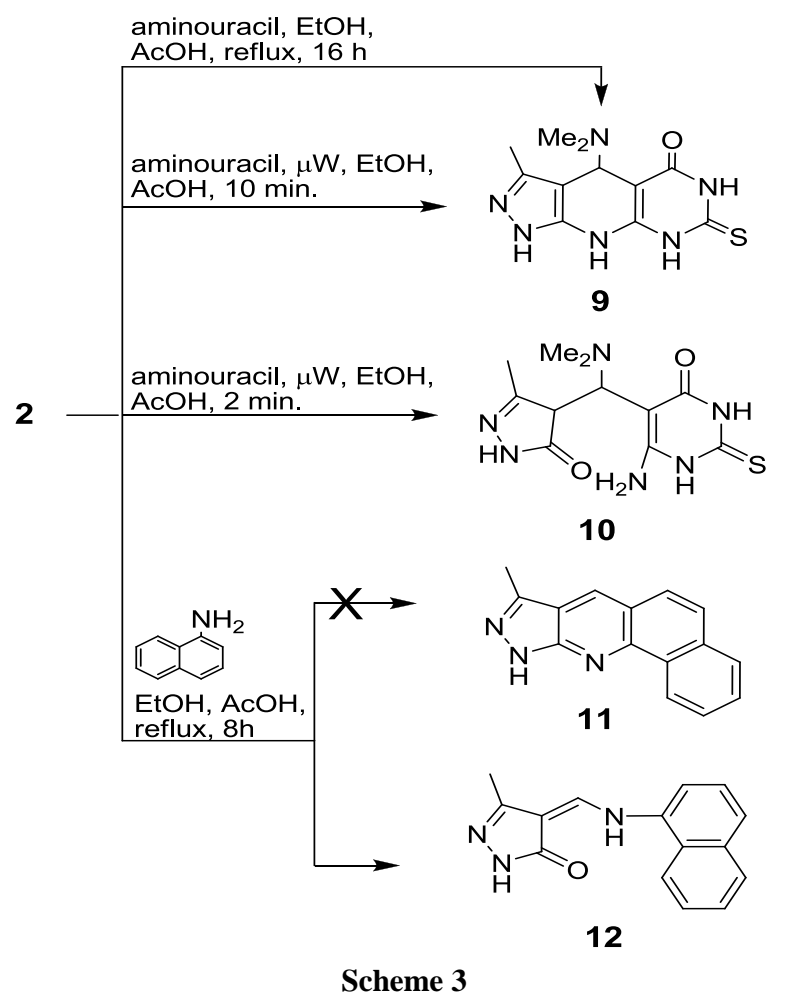

Egypt. J. Chem. 53, No. 5 (2010) 
The previous results encouraged us to study the substitution reactions of compound 2 with heterocyclic C-nucleophiles such as barbituric acid as well as the cycloaddition reactions with hydrazonoyl halides. Compound 2 reacted with barbituric acid derivatives $13 \mathrm{a}, \mathrm{b}$ in ethanol containing hydrochloric acid to form the corresponding substituted adducts $14 \mathrm{a}, \mathrm{b}$, respectively (Scheme 4). On the other hand, the reaction of compound 2 with hydrazonoyl halides was carried out in ethanol containing triethylamine. The latter converted the hydrazonoyl halides into nitrile imine dipolar form which underwent $3+2$ cycloaddition reaction with compound 2 to furnish the corresponding spiro adducts 15a, b (Scheme 4). Enaminones are polyfunctional compounds possessing both electrophilic and nucleophilic properties. Typical electrophilic positions are C-3 (the dimethylaminomethylene center) and $\mathrm{C}-1$ (the carbonyl carbon center) with the reactivity order C-3 > C-1 while a typical nucleophilic position is C-2 ${ }^{(48-51)}$. The regioselectivity of the $3+2$ cycloaddition reaction of compound 2 is in accordance with this hypothesis and excludes the formation of other products. The expected reaction sequence can be explained below (Scheme 4). Primarily, the microanalytical and the spectroscopic data confirmed the structures of the newly synthesized compounds $15 \mathrm{a}, \mathrm{b}$.

$$
\begin{aligned}
& 2 \\
& \prod_{\mathrm{O}}^{\mathrm{RNR}} \\
& \mathrm{EtOH}, \mathrm{HCl} \\
& \text { 13a, } \mathrm{R}=\mathrm{H} \\
& \text { b, } \mathrm{R}=\mathrm{CH} 3 \\
& \text { reflux, } 30 \mathrm{~min} \\
& \mathrm{~N}_{\mathrm{H}}^{\mathrm{N}}=\mathrm{O} \\
& \text { 14a, } R=H \\
& \text { b, } \mathrm{R}=\mathrm{CH} 3
\end{aligned}
$$

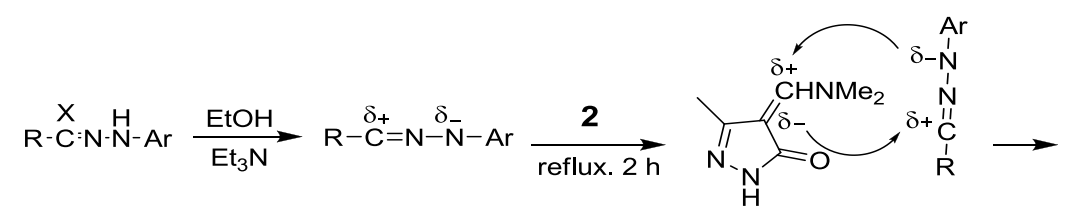

$$
\begin{aligned}
& \sum_{\mathrm{H}}^{\mathrm{Ar}} \\
& \text { 15a, } \mathrm{R}=\mathrm{COCH}_{3}, \mathrm{Ar}=\mathrm{C}_{6} \mathrm{H}_{4}-\mathrm{Cl}-p
\end{aligned}
$$

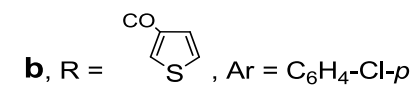

Scheme 4

Egypt. J. Chem. 53, No. 5 (2010) 
Antimicrobial activity

The antimicrobial activity of the newly synthesized compounds 2, 3, 4, 7, 9, 12, and 14a,b against two strains of Gram positive bacteria $\left(\mathrm{G}^{+}\right)$(Bacillus Subtilis and Staphylococcus aureus), two stains of Gram negative bacteria ( $\left.\mathrm{G}^{-}\right)$ (Escherichia coli and Pseudomonas aeroginosa) and two strains of fungi (Aspergillus flavus and candida albicans) was investigated in comparison with Ampicillin and Nystatine. The obtained results are presented in Table 1.

TABLE 1. Antimicrobial potentialities of the tested compounds expressed as size $(\mathrm{mm} / \mathrm{mg}$ sample) of inhibition zone.

\begin{tabular}{|l|c|c|c|c|c|c|c|c|c|c|}
\hline Microorganisms & \multicolumn{10}{|c|}{ Compounds } \\
\hline & $\mathbf{2}$ & $\mathbf{3}$ & $\mathbf{4}$ & $\mathbf{7}$ & $\mathbf{9}$ & $\mathbf{1 2}$ & $\mathbf{1 4 a}$ & $\mathbf{1 4 b}$ & Ampicillin & Nystatine \\
\hline Bacillus subtilis (G+) & 12 & 12 & 13 & 13 & 13 & 15 & 12 & 15 & 18 & - \\
\hline Staphylococcus aureus (G+) & 13 & 12 & 14 & 14 & 13 & 14 & 12 & 13 & 15 & - \\
\hline Escherichia coli (G-) & 13 & 12 & 13 & 13 & 13 & 14 & 12 & 15 & 11 & - \\
\hline Pseudomonas aeroginosa (G-) & 12 & 14 & 12 & 13 & 13 & 15 & 12 & 14 & 19 & - \\
\hline Aspergillus flavus (fungus) & 14 & 14 & 0 & 13 & 0 & 0 & 0 & 0 & - & 12 \\
\hline Candida albicans (fungus) & 11 & 11 & 13 & 12 & 12 & 13 & 12 & 13 & - & 12 \\
\hline
\end{tabular}

In general, all tested compounds were capable of inhibiting the growth of all tested strains. Compounds 12 and $14 \mathrm{~b}$ showed relatively high activity as antibacterial agents. The other compounds showed relatively moderate activity toward all tested strains.

\section{Experimental}

Synthetic methods, analytical and spectral data

Melting points were determined on an electro thermal apparatus (Buchi 535, Switzerland) in an open capillary tube and are uncorrected. IR spectra, expressed in (v, cm-1), were recorded in $\mathrm{KBr}$ pellets on a PA-9721 IR spectrophotometer. 1H-NMR \& 13C-NMR spectra were obtained on a Varian EM-390 $300 \mathrm{MHz}$ spectrometer in DMSO-d6 as solvent, using TMS as an internal reference and chemical shifts $(\delta)$ were expressed in ppm. Mass spectra were recorded on Kratos (75 eV) MS equipment. Elemental analyses were carried out by the Microanalytical Unit at the National Research Center, Giza, Egypt. Microbiological analyses were carried out by the Micro-analytical Center, Faculty of Science, Cairo University, Giza, Egypt.

\section{Synthesis of compounds 2 and 3}

General procedure

A mixture of pyrazolone 1 (4.9 g, $0.05 \mathrm{~mol})$ and DMFDMA (6.6 ml, 0.05 mol) in DMF (30 ml) was heated under reflux for $3 \mathrm{hr}$. The reaction mixture was left to cool at room temperature. The precipitate formed was collected by filtration and crystallized from EtOH to afford $2(80 \%)$. To the filtrate $10 \mathrm{ml}$ of EtOH was added and the solution was left over night. The precipitate was collected by filtration and recrystallized from $\mathrm{DMF} / \mathrm{MeOH}$ to afford $3(20 \%)$. 
4-[(Dimethylamino)methylidene]-5-methyl-2,4-dihydro-3H-pyrazol-3-one (2) Yellow crystals, mp 221-222 ${ }^{\circ} \mathrm{C}$ (DMF). MS (m/z): $153\left(\mathrm{M}^{+}, 100 \%\right)$. IR $(\mathrm{KBr}), \mathrm{v}, \mathrm{cm}^{-1}: 3487(\mathrm{NH}), 2956,2924,2861\left(3 \mathrm{CH}_{3}\right), 1671(\mathrm{C}=\mathrm{O}) .{ }^{1} \mathrm{H}-\mathrm{NMR}$ (300 MHz, DMSO-d6): 1.97 (s, 3H, CH3); 3.24 (s, 3H, N-CH 3 ); 3.75 (s, 3H, N$\left.\mathrm{CH}_{3}\right) ; 7.28(\mathrm{~s}, 1 \mathrm{H}, \mathrm{CH}) ; 10.37(\mathrm{~s}, 1 \mathrm{H}, \mathrm{NH})$. Anal. Calcd. for $\mathrm{C}_{7} \mathrm{H}_{11} \mathrm{~N}_{3} \mathrm{O}: \mathrm{C}, 54.89$; H, 7.24; N, 27.43. Found: C, 54.77; H, 7.08; N, 27.29.

4-[2-(Dimethylamino)-2-(3-methyl-5-oxo-1,5-dihydro-4H-pyrazol-4-ylidene) ethylidene]-5-methyl-2,4-dihydro-3H-pyrazol-3-one (3)

Orange crystals, mp $347-348^{\circ} \mathrm{C}(\mathrm{EtOH}) . \mathrm{MS}(\mathrm{m} / \mathrm{z}): 261\left(\mathrm{M}^{+}, 20 \%\right)$. IR (KBr), $\mathrm{v}, \mathrm{cm}^{-1}: 3452(\mathrm{NH}) ; 2989,2922\left(\mathrm{CH}_{3}\right) ; 1644(\mathrm{C}=\mathrm{O}) .{ }^{1} \mathrm{H}-\mathrm{NMR}(300 \mathrm{MHz}$, DMSO-d $\left.)_{6}\right): 2.21\left(\mathrm{~s}, 6 \mathrm{H}, 2 \mathrm{CH}_{3}\right) ; 2.73(\mathrm{~s}, 3 \mathrm{H}, \mathrm{CH} 3) ; 2.89\left(\mathrm{~s}, 3 \mathrm{H}, \mathrm{CH}_{3}\right) ; 7.36(\mathrm{~s}$, $1 \mathrm{H}, \mathrm{CH}) ; 12.29$ (br, 2H, 2NH). ${ }^{13} \mathrm{C}-\mathrm{NMR}\left(75 \mathrm{MHz}, \mathrm{DMSO}-\mathrm{d}_{6}\right): 17.65$ (two $\mathrm{CH}_{3}$ ); 38.70 (two $\mathrm{CH}_{3}$ ); 98.00 (pyrazolone); 130.12 (pyrazolone C4); 143.20 (ethylene $\mathrm{CH}$ ); 154.80 (two pyrazolone $\mathrm{C} 5$ ); $167.20(\mathrm{C}-\mathrm{N}) ; 168.10$ (two $\mathrm{C}=\mathrm{O}$ ). Anal. Calcd. for $\mathrm{C}_{12} \mathrm{H}_{15} \mathrm{~N}_{5} \mathrm{O}_{2}$ : C, 55.16; H, 5.79; N, 26.80. Found: C, 54.91; H, $5.78 ; \mathrm{N}, 26.42$.

4-Methyl-6H-pyrazolo[4,3-d]isoxazole (4)

Hydroxylamine hydrochloride $(0.01 \mathrm{~mol})$ was added to a solution of pyrazoloenamine $2(0.153 \mathrm{~g}, 0.01 \mathrm{~mol})$ in EtOH $(30 \mathrm{ml})$ containing few drops of $\mathrm{Et}_{3} \mathrm{~N}$. The reaction mixture was heated under reflux for $5 \mathrm{hr}$, cooled and poured into ice/water. The yellow precipitate was collected by filtration and purified by crystallization from $n$-hexane.

Yellow crystals (75\%), mp 194-195 ${ }^{\circ} \mathrm{C}$ (n-hexane). MS (m/z): $123\left(\mathrm{M}^{+}, 88 \%\right)$. IR (KBr), v, cm ${ }^{-1}: 3412(\mathrm{NH}) ; 2920\left(\mathrm{CH}_{3}\right) .{ }^{1} \mathrm{H}-\mathrm{NMR}\left(300 \mathrm{MHz}, \mathrm{DMSO}-\mathrm{d}_{6}\right.$, TMS): 2.25 (s, 3H, $\left.\mathrm{CH}_{3}\right) ; 7.88(\mathrm{~s}, 1 \mathrm{H}, \mathrm{CH}) ; 10.48(\mathrm{~s}, 1 \mathrm{H}, \mathrm{NH})$. Anal. Calcd. for $\mathrm{C}_{5} \mathrm{H}_{5} \mathrm{~N}_{3} \mathrm{O}$ : C, 48.78; H, 4.09; N, 34.13. Found: C, 48.68; H, 4.00; N, 34.15.

3-Methyl-1,5-dihydropyrazolo[3,4-c]pyrazole (5)

To a solution of compound $2(0.153 \mathrm{~g}, 0.001 \mathrm{~mol})$ in $\mathrm{EtOH}(30 \mathrm{ml})$, hydrazine hydrate $(1 \mathrm{ml}, 99 \%)$ and $\mathrm{AcOH}(1 \mathrm{ml})$ were added. The reaction mixture was heated under reflux for $3 \mathrm{hr}$. Compound 5 was filtered as yellow crystals.

Yellow crystals $(78 \%), \mathrm{mp} 309-310^{\circ} \mathrm{C}(\mathrm{EtOH}) . \mathrm{MS}(\mathrm{m} / \mathrm{z}): 122\left(\mathrm{M}^{+}, 30 \%\right)$. IR $(\mathrm{KBr}), \mathrm{v}, \mathrm{cm}^{-1}: 3427(\mathrm{NH}) ; 2926\left(\mathrm{CH}_{3}\right) .{ }^{1} \mathrm{H}-\mathrm{NMR}\left(300 \mathrm{MHz}, \mathrm{DMSO}-\mathrm{d}_{6}\right): 2.23(\mathrm{~s}$, $\left.3 \mathrm{H}, \mathrm{CH}_{3}\right) ; 7.37(\mathrm{~s}, 1 \mathrm{H}, \mathrm{CH}) ; 8.30(\mathrm{~s}, 1 \mathrm{H}, \mathrm{NH}) ; 12.26(\mathrm{~s}, 1 \mathrm{H}, \mathrm{NH}) .{ }^{13} \mathrm{C}-\mathrm{NMR}(75$ MHz, DMSO-d $\left.{ }_{6}\right)$ : $19.44\left(\mathrm{CH}_{3}\right) ; 97.87 ; 148.02$ (C4); 150.11 (C3); 159.25. Anal. Calcd. for $\mathrm{C}_{5} \mathrm{H}_{6} \mathrm{~N}_{4}$ : C, 49.17; H, 4.95; N, 45.88. Found: C, 49.12; H, 4.80; N, 45.73

4-(Dimethylamino)-3-methyl-4,7-dihydro-1H-pyrazolo[3,4-d]pyrimidine-6-thiol (7)

A mixture of pyrazoloenamine $2(0.153 \mathrm{~g}, 0.001 \mathrm{~mol})$ and thiourea $(0.076 \mathrm{~g}$, $0.001 \mathrm{~mol})$ in $\mathrm{EtOH}(30 \mathrm{ml})$ containing $1 \mathrm{ml}$ of $\mathrm{AcOH}$ was heated under reflux for $5 \mathrm{hr}$. The reaction mixture cooled and the solid product formed was filtered off and dried. 
Yellow crystals, mp 251-252 ${ }^{\circ} \mathrm{C}(\mathrm{EtOH})$. MS (m/z): $211\left(\mathrm{M}^{+}, 35 \%\right) 196\left(\mathrm{M}^{+}-\right.$ $\left.\mathrm{CH}_{3}, 35 \%\right)$. IR (KBr), v, cm ${ }^{-1}: 3440(\mathrm{NH}) ; 2961,2919,2851\left(\mathrm{CH}_{3}\right) .{ }^{1} \mathrm{H}-\mathrm{NMR}$ (300 MHz, DMSO-d $)$ ): 1.90 (s, 1H, SH); 2.20 (s, 3H, $\left.\mathrm{CH}_{3}\right) ; 2.21\left(\mathrm{~s}, 6 \mathrm{H}, 2 \mathrm{CH}_{3}\right)$; 7.35 (s, 1H, CH); 9.40 (s, 1H, NH); 12.26 (s, 1H, NH). ${ }^{13} \mathrm{C}-\mathrm{NMR}(75 \mathrm{MHz}$, DMSO-d 6 ): 12.65, 12.86, $13.00\left(3 \mathrm{CH}_{3}\right) ; 66.37(\mathrm{CH}) ; 107.31$ (pyrazole $\mathrm{C} 4$ ); 139.43 (pyrazole C3); 152.45 (pyrazole C5); 163.36 (C-SH). Anal. Calcd. for $\mathrm{C}_{8} \mathrm{H}_{13} \mathrm{~N}_{5} \mathrm{~S}$ : C, 45.48; H, 6.20; N, 33.15. Found: C, 45.35; H, 6.01; N, 32.98.

(E)-2-(Dimethylamino)-2-(3-methyl-5-oxo-1,5-dihydro-4H-pyrazol-4-ylidene)- $N$ phenylethanethioamide (8)

To a solution of pyrazoloenamine $2(0.153 \mathrm{~g}, 0.001 \mathrm{~mol})$ in $\mathrm{EtOH}(30 \mathrm{ml})$ containing $\mathrm{NaOH}(0.04 \mathrm{~g}, 0.001 \mathrm{~mol})$, PhNCS (0.01 mol) was added. The reaction mixture was stirred at room temperature overnight then poured into ice/H2O. The solid product formed was collected by filtration.

Yellow crystals, mp $158-159^{\circ} \mathrm{C}$ (benzene and n-hexane). MS $(\mathrm{m} / \mathrm{z}): 288\left(\mathrm{M}^{+}\right.$, 20\%). ${ }^{1} \mathrm{H}-\mathrm{NMR}\left(300 \mathrm{MHz}, \mathrm{DMSO}-\mathrm{d}_{6}\right): 2.17\left(\mathrm{~s}, 3 \mathrm{H}, \mathrm{CH}_{3}\right) ; 2.33\left(\mathrm{~s}, 6 \mathrm{H}, 2 \mathrm{CH}_{3}\right)$; 6.97-7.71 (m, 5H, aromatic protons); 9.68 (br, s, $1 \mathrm{H}, \mathrm{NH}) ; 12.29$ (br, s, 1H, NH). ${ }^{13} \mathrm{C}-\mathrm{NMR}\left(75 \mathrm{MHz}, \mathrm{DMSO}-\mathrm{d}_{6}\right): 17.78\left(\mathrm{CH}_{3}\right) ; 38.30\left(2 \mathrm{CH}_{3}, \mathrm{NMe}\right) ; 38.38$ (pyrazole C4); 112.21 (pyrazole C3), [124.50, 125.13, 128.60, 139.42] (six aromatic carbons); 155.61 (C-N, linker); 168.00 (pyrazole $\mathrm{C}=\mathrm{O}), 188.23(\mathrm{C}=\mathrm{S})$. Anal. Calcd. for $\mathrm{C}_{14} \mathrm{H}_{16} \mathrm{~N}_{4} \mathrm{OS}$ : C, 58.31; H, 5.59; N, 19.43; S, 11.12. Found : C, 58.01; H, 5.35; N, 19.21; S, 11.00.

4-(Dimethylamino)-3-methyl-7-thioxo-1,4,6,7,8,9-hexahydro-5H-pyrazolo $\left[4^{\prime}, 3^{\prime}: 5,6\right]$ pyrido[2,3-d]pyrimidin-5-one (9)

Method A: Conventional heating

A mixture of pyrazoloenamine $2(0.153 \mathrm{~g}, 0.001 \mathrm{~mol})$ and thiouracil $(0.143 \mathrm{~g}$, $0.001 \mathrm{~mol})$ in DMF $(20 \mathrm{ml})$ containing AcOH $(1 \mathrm{ml})$ was heated under reflux for $16 \mathrm{hr}$. The reaction mixture was left to cool and poured onto ice/H2O. The solid product formed was collected by filtration.

\section{Method B: Microwave heating}

An opened Erlenmeyer flask, containing a mixture of pyrazoloenamine 2 $(0.153 \mathrm{~g}, 0.001 \mathrm{~mol})$ and thiouracil $(0.143 \mathrm{~g}, 0.001 \mathrm{~mol})$ in $3 \mathrm{ml}$ DMF was placed in domestic microwave oven and irradiated for $10 \mathrm{~min}$. The reaction mixture was left to cool at room temperature. The resulting product was triturated with nhexane. The formed precipitate was collected by filtration and crystallized from the suitable solvent.

Yellow solid (13\% and $58 \%$ for conventional heating and microwave irradiation, respectively), $\mathrm{mp}>350^{\circ} \mathrm{C}(\mathrm{DMF}) . \mathrm{MS}(\mathrm{m} / \mathrm{z}): 278\left(\mathrm{M}^{+}, 68 \%\right)$. IR $(\mathrm{KBr}), \mathrm{v}, \mathrm{cm}^{-1}$ : $3449(\mathrm{NH}) ; 2964,2889\left(\mathrm{CH}_{3}\right) ; 1619(\mathrm{C}=\mathrm{O}) .{ }^{1} \mathrm{H}-\mathrm{NMR}(300 \mathrm{MHz}$, DMSO-d $)_{6}$ : 2.09 (s, 3H, $\left.\mathrm{CH}_{3}\right) ; 2.51\left(\mathrm{~s}, 6 \mathrm{H}, 2 \mathrm{CH}_{3}\right) ; 5.04(\mathrm{~s}, 1 \mathrm{H}, \mathrm{CH}) ; 7.21(\mathrm{~s}, 1 \mathrm{H}$, exchangeable NH); $8.17(\mathrm{~s}, 1 \mathrm{H}, \mathrm{NH}) ; 8.82(\mathrm{~b}, \mathrm{H}, \mathrm{NH}) ; 11.45(\mathrm{~s}, 1 \mathrm{H}, \mathrm{NH}) .{ }^{13} \mathrm{C}-$ NMR (75 MHz, DMSO-d $\left.)_{6}\right) 17.40\left(\mathrm{CH}_{3}\right) ; 38.37\left(2 \mathrm{C}, \mathrm{N}\left(\mathrm{CH}_{3}\right) 2\right) ; 45.60,88.60$,

Egypt. J. Chem. 53, No. 5 (2010) 
103.00, 142.70, 156.10, 162.70, $167.21(\mathrm{C}=\mathrm{O}), 168.00(\mathrm{C}=\mathrm{S})$. Anal. Calcd. for $\mathrm{C}_{11} \mathrm{H}_{14} \mathrm{~N}_{6} \mathrm{OS}$ : C, 47.47; H, 5.07; N, 30.19; S, 11.52. Found: C, 47.25; H, 4.89; N, 30.02; S, 11.41 .

6-Amino-5- [(dimethylamino) (3-methyl-5-oxo-4,5-dihydro-1H-pyrazol-4-yl) methyl]2-thioxo-2,3-dihydropyrimidin-4(1H)-one (10)

An opened Erlenmeyer flask, containing a mixture of pyrazoloenamine 2 $(0.153 \mathrm{~g}, 0.001 \mathrm{~mol})$ and thiouracil $(0.143 \mathrm{~g}, 0.001 \mathrm{~mol})$ dissolved in DMF $(2 \mathrm{ml})$, was placed in domestic microwave oven and irradiated for $2 \mathrm{~min}$. The reaction mixture was left to cool at room temperature. The resulting product was triturated with n-hexane. The formed solid was collected by filtration.

Red crystals $(63 \%), \mathrm{mp} 288-290^{\circ} \mathrm{C}(\mathrm{EtOH}) . \mathrm{MS}(\mathrm{m} / \mathrm{z}): 296\left(\mathrm{M}^{+}, 6 \%\right)$. IR $(\mathrm{KBr}), \mathrm{v}, \mathrm{cm}^{-1}: 3450(\mathrm{NH}) ; 2996,2896\left(\mathrm{CH}_{3}\right) ; 1645,1615(2 \mathrm{C}=\mathrm{O}) .{ }^{1} \mathrm{H}-\mathrm{NMR}$ (300 MHz, DMSO-d $)_{6}: 2.22\left(\mathrm{~s}, 3 \mathrm{H}, \mathrm{CH}_{3}\right) ; 2.64\left(\mathrm{~s}, 6 \mathrm{H}, 2 \mathrm{CH}_{3}\right) ; 4.71(\mathrm{~d}, 1 \mathrm{H}$, pyrazole $\mathrm{CH}) ; 5.04(\mathrm{~d}, 1 \mathrm{H}$, methylidene $\mathrm{CH}) ; 6.69(\mathrm{~s}, 1 \mathrm{H}$, exchangeable $\mathrm{NH})$; 7.37 (s, 1H, NH); 9.11 (br, 2H, $\left.\mathrm{NH}_{2}\right) ; 11.30(\mathrm{~s}, 1 \mathrm{H}, \mathrm{NH})$. Anal. Calcd. for $\mathrm{C}_{11} \mathrm{H}_{16} \mathrm{~N}_{6} \mathrm{O}_{2} \mathrm{~S}: \mathrm{C}, 44.58 ; \mathrm{H}, 5.44 ; \mathrm{N}, 28.36 ; \mathrm{S}, 10.82$. Found: C, 44.45; H, 5.30; N, 28.18; S, 10.71 .

5-Methyl-4-[(naphthalen-1-ylamino)methylidene]-2,4-dihydro-3H-pyrazol-3-one (12)

A mixture of pyrazoloenamine $2(0.153 \mathrm{~g}, 0.001 \mathrm{~mol})$ and $\alpha$-naphthylamine $(0.143 \mathrm{~g}, 0.001 \mathrm{~mol})$ in absolute $\mathrm{EtOH}(30 \mathrm{ml})$ and $\mathrm{AcOH}(1 \mathrm{ml})$ was heated under reflux for $8 \mathrm{hr}$. The reaction mixture was left to cool. The solvent was evaporated under vacuum. The residue was triturated with n-hexane and the solid formed was collected by filtration.

Brown solid (69\%), mp 282-283 ${ }^{\circ} \mathrm{C}(\mathrm{EtOH}) . \mathrm{MS}(\mathrm{m} / \mathrm{z}): 251\left(\mathrm{M}^{+}, 100 \%\right)$. IR $(\mathrm{KBr}), \mathrm{v}, \mathrm{cm}^{-1}: 3431(\mathrm{NH}) ; 2922\left(\mathrm{CH}_{3}\right) ; 1671(\mathrm{C}=\mathrm{O}) .1 \mathrm{H}-\mathrm{NMR}(300 \mathrm{MHz}$, DMSO-d $\left.{ }_{6}\right): 2.21\left(\mathrm{~s}, 3 \mathrm{H}, \mathrm{CH}_{3}\right) ; 7.39(\mathrm{~s}, 1 \mathrm{H}, \mathrm{CH}) ; 7.59-8.09$ (m, 7H, naphthalene); $8.78\left(\mathrm{~s}, 1 \mathrm{H}\right.$, exchangeable NH); $11.19(\mathrm{~s}, 1 \mathrm{H}, \mathrm{NH}) .{ }^{13} \mathrm{C}-\mathrm{NMR}(75 \mathrm{MHz}, \mathrm{DMSO}$ $\mathrm{d}_{6}$ ): $12.71\left(\mathrm{CH}_{3}\right) ; 102.52$ (pyrazole $\mathrm{C} 3$ ); 112.51, 118.72, 120.72, 123.00, 124.60, 125.66, 126.24, 128.32, 133.82, 134.37 (naphthalene carbons); 145.45 (methylidene carbon); 147.45 (pyrazole $\mathrm{C} 5) ; 169.01(\mathrm{C}=\mathrm{O})$. Anal. Calcd. for $\mathrm{C}_{15} \mathrm{H}_{13} \mathrm{~N}_{3} \mathrm{O}$ : C, 71.70; H, 5.21; N, 16.72. Found: C, 71.55; H, 5.00; N, 16.35.

Synthesis of compounds $14 a, b$

General procedure

A mixture of pyrazoloenamine $2(0.153 \mathrm{~g}, 0.001 \mathrm{~mol})$ and barbituric acid (13a and $13 \mathrm{~b})(0.001 \mathrm{~mol})$ in $\mathrm{EtOH}(25 \mathrm{ml})$ containing $\mathrm{HCl}(1 \mathrm{ml})$ was heated under reflux for $30 \mathrm{~min}$. The reaction mixture was left to cool. The solid product formed was collected by filtration.

5-[(3-Methyl-5-oxo-1,5-dihydro -4H-pyrazol-4-ylidene) methyl] pyrimidine-2,4,6 (1H,3H,5H)-trione (14a)

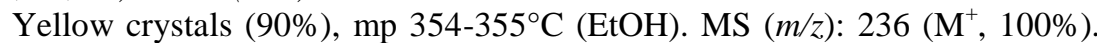
IR (KBr), v, cm ${ }^{-1}: 3450(\mathrm{NH}) ; 2960(\mathrm{M} / \mathrm{Z} \%) ; 1744,1683,1638,1618(\mathrm{C}=\mathrm{O})$. 
${ }^{1} \mathrm{H}-\mathrm{NMR}$ (300 MHz, DMSO-d $\left.{ }_{6}\right): 2.19$ (s, 3H, M/Z \%); 3.46 (d, 1H, CH pyrimidine); $8.07(\mathrm{~d}, 1 \mathrm{H}, \mathrm{CH}) ; 11.26(\mathrm{~s}, 2 \mathrm{H}, 2 \mathrm{NH}) ; 11.57(\mathrm{~s}, 1 \mathrm{H}, \mathrm{NH}) .{ }^{13} \mathrm{C}-\mathrm{NMR}$ (75 MHz, DMSO-d ${ }_{6}$ ): $17.52\left(\mathrm{CH}_{3}\right) ; 48.22$ (pyrimidine $\mathrm{C} 5$ ); 129.81 (pyrazole $\mathrm{C} 4$ ); 139.33 (methylene $\mathrm{CH}$ ); 155.63 (pyrazole $\mathrm{C} 3$ ); 157.24 (pyrimidine $\mathrm{C} 2$ ); 168.00 (pyrazole $\mathrm{C} 5$ ); 170.70 (2C, pyrimidine $\mathrm{C} 4$ and $\mathrm{C} 6$ ). Anal. Calcd. for $\mathrm{C}_{9} \mathrm{H}_{8} \mathrm{~N}_{4} \mathrm{O}_{4}: \mathrm{C}, 45.77 ; \mathrm{H}, 3.41 ; \mathrm{N}, 23.72$. Found: $\mathrm{C}, 45.54 ; \mathrm{H}, 3.22 ; \mathrm{N}, 23.31$.

1,3-Dimethyl-5- [(3-methyl-5-oxo-1,5-dihydro-4H-pyrazol-4-ylidene) methyl] pyrimidine2,4,6(1H,3H,5H)-trione $(14 b)$

Yellow crystals $(95 \%), \mathrm{mp} 296-297^{\circ} \mathrm{C}(\mathrm{EtOH}) . \mathrm{MS}(\mathrm{m} / \mathrm{z}): 264\left(\mathrm{M}^{+}, 25 \%\right)$. IR $(\mathrm{KBr}), \mathrm{v}, \mathrm{cm}^{-1}: 3430(\mathrm{NH}) ; 2961,2920,2853\left(\mathrm{CH}_{3}\right) ; 1779,1728,1665,1633$ $(\mathrm{C}=\mathrm{O}) .{ }^{1} \mathrm{H}-\mathrm{NMR}\left(300 \mathrm{MHz}, \mathrm{DMSO}-\mathrm{d}_{6}\right): 2.21\left(\mathrm{~s}, 3 \mathrm{H}, \mathrm{CH}_{3}\right) ; 2.46\left(\mathrm{~s}, 6 \mathrm{H}, \mathrm{CH}_{3}\right)$; $3.47(\mathrm{~d}, 1 \mathrm{H}, \mathrm{CH}) 8.07(\mathrm{~d}, 1 \mathrm{H}, \mathrm{CH}) 11.55(\mathrm{~s}, 1 \mathrm{H}, \mathrm{NH})$. Anal. Calcd. for $\mathrm{C}_{11} \mathrm{H}_{12} \mathrm{~N}_{4} \mathrm{O}_{4}$ : C, 50.00; H, 4.58; N, 21.20. Found: C, 49.88; H, 4.30; N, 21.01.

Synthesis of compounds $15 a, b$

General procedure

To a stirred solution of hydrazonoyl halide $(0.01 \mathrm{~mol})$ in $\mathrm{EtOH}$ containing Et3N $(0.01 \mathrm{~mol})$, pyrazoloenamine $2(1.53 \mathrm{~g}, 0.01 \mathrm{~mol})$ was added. The reaction mixture was stirred for $1 \mathrm{hr}$ and then heated under reflux for $2 \mathrm{hr}$. The reaction mixture was left to cool and poured onto ice $/ \mathrm{H}_{2} \mathrm{O}$. The solid product formed was collected by filtration and crystallized from $\mathrm{EtOH}$.

6-Acetyl-8-(4-chlorophenyl)-9- (dimethylamino) -4- methyl-2,3,7,8tetraazaspiro[4.4] nona-3,6-dien-1-one (15a)

Brown crystals $(55 \%), \mathrm{mp} 169-170^{\circ} \mathrm{C}(\mathrm{EtOH}) . \mathrm{MS}(\mathrm{m} / \mathrm{z}): 347\left(\mathrm{M}^{+}\right)$. IR $(\mathrm{KBr})$, $\mathrm{v}, \mathrm{cm}^{-1}: 2978,2928,1890\left(\mathrm{CH}_{3}\right) ; 1664,1627(2 \mathrm{C}=\mathrm{O}) .{ }^{1} \mathrm{H}-\mathrm{NMR}(300 \mathrm{MHz}$, DMSO-d $\left.)_{6}\right): 1.97\left(\mathrm{~s}, 3 \mathrm{H}, \mathrm{CH}_{3}\right) ; 2.20\left(\mathrm{~s}, 3 \mathrm{H}, \mathrm{CH}_{3}\right) ; 2.33\left(\mathrm{~s}, 6 \mathrm{H}, 2 \mathrm{CH}_{3}\right) ; 4.11(\mathrm{~s}, 1 \mathrm{H}$, $\mathrm{CH})$; 7.37-7.73 (m, 5H, aromatic protons and NH). 13C-NMR (75 MHz, DMSO$\left.\mathrm{d}_{6}\right): 14.91\left(\mathrm{CH}_{3}\right), 19.33\left(\mathrm{CH}_{3}\right), 36.00\left(2 \mathrm{CH}_{3}\right) ; 52.00$ (spiro carbon); 70.52, 117.00 (pyrazole C5); 113.00, 122.22, 129.72, 141.61 (aromatic carbons); 155.00 (C6); 155.66 (C4); 177.00, $200.00(2 \mathrm{C}=\mathrm{O})$. Anal. Calcd. for $\mathrm{C}_{16} \mathrm{H}_{18} \mathrm{ClN}_{5} \mathrm{O}_{2}$ : C, 55.25; H, 5.22; Cl, 10.19; N, 20.14. Found: C, 55.05; H, 5.00; Cl, 9.91; N, 19.95.

8-(4-Chlorophenyl)-9-(dimethylamino)-4-methyl-6- (thiophen-3-ylcarbonyl) 2,3,7,8-tetraazaspiro[4.4]nona-3,6-dien-1-one (15b)

Yellow crystals $(62 \%), \mathrm{mp} 180-181^{\circ} \mathrm{C}(\mathrm{EtOH})$. MS $(\mathrm{m} / \mathrm{z}): 415\left(\mathrm{M}^{+}\right)$. IR $(\mathrm{KBr}), \mathrm{v}, \mathrm{cm}^{-1}: 3430(\mathrm{NH}) ; 2978,2928\left(\mathrm{CH}_{3}\right) ; 1664,1627(2 \mathrm{C}=\mathrm{O}) .{ }^{1} \mathrm{H}-\mathrm{NMR}$ (300 MHz, DMSO-d $)_{6}$ : $2.28\left(\mathrm{~s}, 3 \mathrm{H}, \mathrm{CH}_{3}\right) ; 2.34\left(\mathrm{~s}, 6 \mathrm{H}, 2 \mathrm{CH}_{3}\right) ; 4.20(\mathrm{~s}, 1 \mathrm{H}, \mathrm{CH})$; $7.34(\mathrm{~m}, 2 \mathrm{H}$, aromatic proton); $7.50(\mathrm{~m}, 2 \mathrm{H}$, aromatic protons); 8.07-8.15 (m, $3 \mathrm{H}$, thiophene protons); $11.18(\mathrm{~s}, 1 \mathrm{H}, \mathrm{NH})$. Anal. Calcd. for $\mathrm{C}_{19} \mathrm{H}_{18} \mathrm{ClN}_{5} \mathrm{O}_{2} \mathrm{~S}: \mathrm{C}$, 54.87; H, 4.36; Cl, 8.52; N, 16.84; S, 7.71. Found: C, 54.55; H, 4.20; Cl, 8.32; N, $16.65 ; \mathrm{S}, 7.50$.

Bioassay

A filter paper sterilized disc saturated with measured quantity of the sample is placed on plate containing solid bacterial medium (nutrient agar broth) or fungal Egypt. J. Chem. 53, No. 5 (2010) 
medium (Dox's medium) which has been heavily seeded with spore suspension of the tested organism. After inoculation, the diameter of the clear zone of inhibition surrounding the sample is taken as a measure of the inhibitory power of the sample against test organism ${ }^{(52-54)}$.

\section{References}

1. Bazigir, A., Khanposhtani, M.M. and Sooki, A.A., Bioorg. Med. Chem. Lett., 18, 5800 (2008).

2. Bondoch, S., Rabie, R., Etman, H.A. and A., F.A., J. Med. Chem., 43, 2122 (2008).

3. Gürsoy, A., Demirayak, S., Capan, G., Erol, K. and Vural, K., Eur. J. Med. Chem., 35, 359 (2000).

4. Goel, A. and Madan, A.K., J. Chem. Inf. Comput. Sci., 35, 510 (1995).

5. Ranatunge, R.R., Augustyniak, M., Bandarage, U.K., Earl, R.A., Ellis, J.L., Garvey, D.S., Janero, D.R., Letts, L.G., Martino, A.M., Murty, M.G., Richardson, S.K., Schroeder, J.D., Shumway, M.J., Tam, S.W., Trocha, A.M. and Young, D.V., J. Med. Chem., 47, 2180 (2004).

6. Fancelli, D., Moll, J., Varasi, M., Bravo, R., Artico, R., Berta, D., Bindi, S., Cameron, A., Candiani, I., Cappella, P., Carpinelli, P., Croci, W., Forte, B., Giorgini, M.L., Klapwijk, J., Marsiglio, A., Pesenti, E., Rocchetti, M., Roletto, F., Severino, D., Soncini, C., Storici, P., Tonani, R., Zugnoni, P. and Vianello, P., J. Med. Chem., 49, 7247 (2006).

7. Gökhan-Kelekçia, N., Yabanoğlu, S., Küpeli, E., Salgın, U., Özgen, Ö., Uçar, G., Yeşilada, E., Kendi, E., Yeşilada, A. and Bilgin, A.A., Bioorg Med Chem., 15, 5775 (2007).

8. Pevarello, P., Brasca, M.G., Amici, R., Orsini, P., Traquandi, G., Corti, L., Piutti, C.; Sansonna, P., Villa, M., Pierce, B.S., Pulici, M., Giordano, P., Martina, K., Fritzen, E.L., Nugent, R.A., Casale, E., Cameron, A., Ciomei, M., Roletto, F., Isacchi, A., Fogliatto, G., Pesenti, E., Pastori, W., Marsiglio, A., Leach, K.L., Clare, P.M., Fiorentini, F., Varasi, M., Vulpetti, A. and Warpehoski, M.A., J. Med. Chem., 47, 3367 (2004).

9. Baraldi, P.G., Balboni, G., Pavani, M.G., Spalluto, G., Tabrizi, M.A., Clercq, E.D., Balzarini, J., Bando, T., Sugiyama, H. and Romagnoli, R., J. Med. Chem., 44, 2536 (2001).

10. Franchini, M.C., Bonini, B.F., Camaggi, C.M., Gentili, D., Pession, A., Rani, M. and Strocchi, E., European Journal of Medicinal Chemistry, 45, 2024 (2010).

11. Sullivan, T.J., Truglio, J.J., Boyne, M.E., Novichenok, P., Zhang, X., Stratton, C.F., Li, H.-J., Kaur, T., Amin, A., Johnson, F., Slayden, R.A., Kisker, C. and Tonge, P.J., ACS Chem. Biol., 1, 43 (2006).

12. El-Emary, T.I. and Banhite, E.A., Pharmazi, 54, 106 (1999). 
13. Castagnolo, D., Manetti, F., Radi, M., Bechi, B., Pagano, M., De Logu, A., Meleddu, R.; Saddi, M. and Botta, M., Bioorg. Med. Chem. Lett., 17, 5716 (2009).

14. Heller, S.T. and Natarajan, S.R., Org Lett., 8, 2675 (2006).

15. Kakiuchi, Y., Sasaki, N., Mieko Satoh-Masuoka a, b., Murofushi, H. and Murakami-Murofushi, K., Biochem. Biophys. Res. Commun., 320, 1351 (2004).

16. Lin, R., Chiu, G., Yu, Y., Connolly, P.J., Li, S., Lu, Y., Adams, M., FuentesPesquers, A.R., Emanuel, S.L. and Greenberger, L.M., Bioorg Med Chem Lett., 17, 4557 (2007).

17. Watanabe, T., Yuki, S., Egawa, M. and Nishi, H., J. Pharmacol. Exp. Ther., 268 1597 (1994).

18. Kawai, H., Nakai, H., Suga, M., Yuki, S., Watanabe, T. and Saito, K.I., J. Pharmacol. Exp. Ther., 281, 921 (1997).

19. Wu, T.-W., Zeng, L.-H., Wu, J. and Fung, K.P., Life Sci., 71, 2249 (2002).

20. Himly, M., Jahn-Schmid, B., Pittertschatscher, K., Bohle, B., Grubmayr, K., Ferreira, F., Ebner, H. and Ebner, C., J Allergy Clin Immunol., 111, 882 (2003).

21. Barba, O. and Jones, L.H., WO/2004/029042; (2004).

22. DeWald, H.A., Lobbestael, S. and Poschel, B.P.H., J. Med. Chem., 24, 982 (1981).

23. Penning, T.D., Talley, J.J., Bertenshaw, S.R., Carter, J.S., Collins, P.W., Docter, S.; Graneto, M.J., Lee, L.F., Malecha, J.W., Miyashiro, J.M., Rogers, R.S., Rogier, D.J., Yu, S., Anderson, G.D., Burton, E.G., Cogburn, J.N., Gregory, S.A., Koboldt, C.M., Perkins, W.E., Seibert, K., Veenhuizen, A.W., Zhang, Y.Y. and Isakson, P.C., J. Med. Chem., 40, 1347 (1997).

24. Terrett, N.K., Bell, A.S.; Brown, D. and Ellis, P., Bioorg. Med. Chem. Lett., 6, 1819 (1996).

25. Rinaldi-Cormona, M., Barth, F., Héaulme, M., Shire, D., Calandra, B., Congy, C., Martinez, S., Maruani, J., Néliat, G., Caput, D., Ferrara, P., Soubrié, P., Brelière, J.C. and Fur, G.L., FEBS Lett., 350, 240 (1994).

26. Deng, X. and Mani, N.S., Org Lett., 8, 3505 (2006).

27. Rashad, A.E., Hegab, M.I., Abdel-Megeid, R.E., Mick, J.A. and Abdel-Megied, F.M.E., Bioorg. Med. Chem., 16, 7102 (2008).

28. Saikia, A. Barthakur, M.G., Borthakur, M., Saikia, C.J., Bora, U. and Bouruah, R.C., Tetrahedron Lett., 47, 43 (2006).

29. Karci, F. and Demircali, A., Dyes Pigm. 74, 288 (2007).

30. Csunderlik, C.; Bercean, V., Peter, F. and Badea, V., ARKIVOC ii, 133 (2002).

Egypt. J. Chem. 53, No. 5 (2010) 
31. Vicentini, C.B., Guccione, S.; Giurato, L., Ciaccio, R., Mares, D. and Forlani, G., J. Agric. Food Chem., 53, 3848 (2005).

32. Waldrep, T.W., Beck, J.R., Lynch, M.P. and Wright, F.L., J. Agric. Food Chem., 38, 541 (1990).

33. Siddal, T.L., Ouse, D.G., Benko, Z.L., Garvin, G.M., Jackson, J.L., McQuiston, J.M., Ricks, M.J., Thibault, T.D., Turner, J.A., VanHeertum, J.C. and Weimer, M.R., Pest Manage Sci., 58, 1175 (2002).

34. Mohamed, N.R., El-Saidi, M.M.T., Ali, Y.M. and Elnagdi, M.H., Bioorg. Med. Chem., 15, 6227 (2007).

35. Mohamed, N.R., El-Saidi, M.M.T., Ali, Y.M. and Elnagdi, M.H., J. Het. Chem., 44, 1333 (2007).

36. Stanovnik, B. and Svete, J., Chemical Reviews, 104, 2433-2480 (2004).

37. Khan, R., Uddin, M.I., Alam, M.S., Hossain, M.M. and Islam, M.R., Bangladesh .J Pharmacol, 3, 27 (2008).

38. Mašič, L.P. and Kikelj, D., Tetrahedron Lett., 41, 5589 (2000).

39. Powell, D., Gopalsamy, A., Wang, Y.D., Zhang, N., Miranda, M., McGinnis, J.P. and Rabindran, S.K., Bioorg. Med. Chem. Lett., 17, 1641 (2007).

40. Braibante, M.E.F., Braibante, H.T.S., Costa, C.C. and Martins, D.T.B., Tetrahedron Lett., 43, 8079 (2002).

41. Pawar, R.A. and Patil, A.A., Indian J. Chem., Sect. B: Org. Chem. Incl. Med. Chem., 33B, 156 (1994).

42. Abd El-Latif, F.M., Asian J. Chem., 5, 184 (1993).

43. Abd El-Latif, F.M., Asian J. Chem., 71, 631 (1994).

44. Holzer, W. and Hahn, K., J. Heterocycl. Chem., 40, 303 (2003).

45. Mohareb, R.M. and Hana, H.Y., Acta Pharm., 58, 29 (2008).

46. Bose, A.K., Manhas, M.S., Ghosh, M., Shah, M., Raju, V.S., Bari, S.S., Newaz, S.N., Banki, B.K., Chaudhary, A.G. and Barakat, K.J., J. Org. Chem., 56, 6968 (1991).

47. Bose, A.K., Banik, B.K., Lavlinskaia, N., Jayaraman, M. and Manhas, M.S., Chemtech., 18 (1997).

48. Stanovnik, B., Molecules, 1, 123 (1996).

49. Stanovnik, B., J. Heterocycl. Chem., 36, 1581 (1999).

50. Stanovnik, B. and Svete, J., Synlett, 1077 (2000). 
51. Svete, J., J. Heterocycl. Chem., 39, 437 (2002).

52. Grayer, R.J. and Harbone, J.B., Phytochemistry, 37, 19 (1994).

53. Muanza, D.N., Kim, B.W., Euler, K.L. and Williams, L., Int J Pharmacog., 32, 337 (1994).

54. Irob, O.N., Moo-Young, M. and Anderson, W.A., Int. J. Pharmacog., 34, 87 (1996).

(Received 14 /2 / 2010; accepted $4 / 11 / 2010$

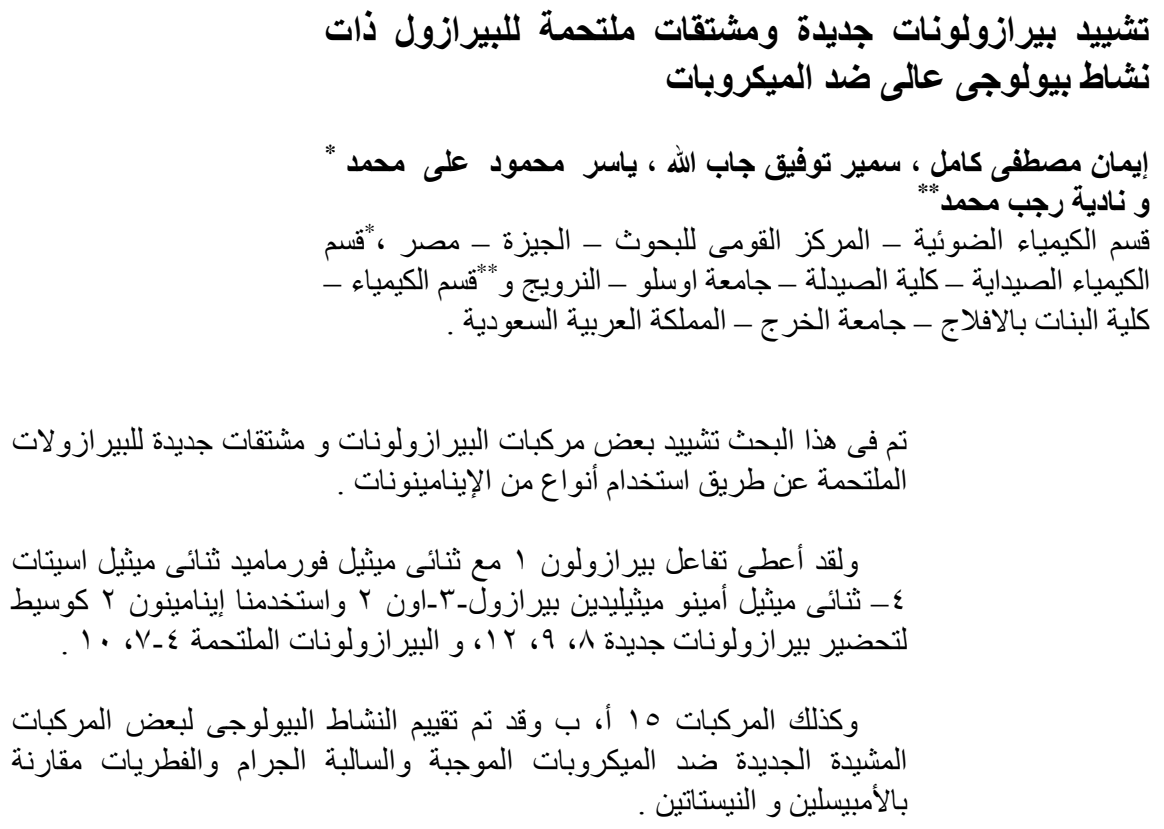

